Note de recherche

\title{
Evaluation de l'activité hémagglutinante des lectines des graines de trois espèces de Cucurbitaceae couramment consommées en Côte d'Ivoire
}

\author{
Alassane Meite *, Koffi G. Kouame \& Atté M. Offoumou \\ Laboratoire Nutrition et Pharmacologie, UFR Biosciences, Université de Cocody Abidjan Côte d'Ivoire, 22 BP 582 Abidjan 22 \\ ${ }^{*}$ Auteur pour les correspondances (E-mail : almeite@yahoo.fr) \\ Recu le 11-07-2006, accepté le 15-02-2008.
}

\section{Résumé}

L'activité hémagglutinante des lectines est testée sur les graines de trois espèces de courges (Cucurbitaceae) Citrullus lanatus (CL), Citrullus sp. (Csp), Cucumeropsis edulis (CE) de consommation courante en Côte d'Ivoire (Afrique de l'Ouest) et comparée à celle des graines de haricot Phaseolus vulgaris (PV).

Les extraits de toutes les graines de courges montrent une activité hémagglutinante faible (4 à 128) par rapport à celle des graines de haricot (512 à 1024). Les hématies de lapin sont les plus réactives vis-à-vis des extraits bruts des graines de courges et de haricot. En plus, le traitement trypsique augmente la réactivité de ces mêmes hématies.

La fraction protéique $\mathrm{F} 5$, obtenue par précipitation au sulfate d'ammonium à $95 \%$ de saturation, de l'extrait brut de Csp présente l'activité hémagglutinante la plus élevée (512).

Le traitement thermique à $100^{\circ} \mathrm{C}$ pendant 15 minutes réduit significativement l'activité hémagglutinante des lectines des extraits bruts des graines de courges et de haricot ; le même traitement appliqué pendant 30 minutes abouti à une inactivation totale des lectines de toutes ces graines.

Mots clés: Activité hémagglutinante, lectines, courges, cucurbitaceae

\begin{abstract}
Evaluation of lectin hemagglutinating activity of three species of Cucurbitaceae seeds frequently consumed in Côte d'lvoire
\end{abstract}

Lectin hemagglutinating activity has been assessed on the seeds of three species of Cucurbitaceae: Citrullus lanatus (CL), Citrullus sp. (Csp) and Cucumeropsis edulis (CE) of current consumption in Côte d'lvoire (West Africa), and compared with the seeds of haricot beans Phaseolus vulgaris (PV). The extracts of all the seeds of Cucurbitaceae exhibit low levels of lectin activity (4 to 128), compared to those of beans (512 to 1024). The red blood cells of rabbit exhibit the highest reactivity both with Cucurbitaceae and beans seed crude extract. Moreover the treatment with trypsin enhances these same red blood cells reactivity.

The highest levels of lectin activity have been measured on the protein fraction (F5) obtained by precipitation of the raw extract of Csp with the ammonium sulphate solution to $95 \%$ of saturation.

The thermal treatment at $100{ }^{\circ} \mathrm{C}$ temperature for 15 minutes significantly reduce the levels of lectin activity of both seeds extracts of Cucurbitaceae and beans; the same treatment applied for 30 minutes results in total inactivation of the lectins of all these seeds.

Key words: hemagglutinating activity, lectin, Cucurbitaceae 


\section{Introduction}

La malnutrition protéino-calorique est très fréquente dans les pays du tiers monde (surtout en zones rurales) (Dos Santos et Damon, 1987). Cette sous-nutrition protéique, qui favorise des infections, peut-être due à plusieurs facteurs dont le principal est l'insuffisance de protéines disponibles pour une population en croissance rapide (Dos Santos et Damon, 1987).

Les ressources végétales, à cause de leur disponibilité et leur faible coût par rapport aux protéines animales, peuvent constituer une solution dans l'amélioration de la qualité nutritionnelle des populations du tiers monde. Pour que cette solution soit efficace, des connaissances sur les données agronomiques (robustesse de la plante, rendement) mais également les conclusions d'études strictement nutritionnelles sont nécessaires.

Tout comme certaines graines de légumineuses (haricot, soja), des graines alimentaires de consommation courante en Afrique telles que celles de courges (Cucurbitaceae) (Aké Assi, 1984; Kiki-M'vouaka et al., 1988) sont également de sources végétales riches en protéines et peuvent ainsi constituer une solution à la malnutrition protéino-calorique. En effet, les travaux de Quenum et Kouadio (1986) indiquent que les teneurs en protéines des graines de courges des cultivars exploités en Côte d'Ivoire varient de $32 \%$ à $48 \%$ de matière sèche. De même, Camara (1996) montre que les graines de Citrullus lanatus renferment $39,40 \%$ de protéines. D'autre part, de telles plantes offrent l'avantage d'être connues à la fois des cultivateurs et des ménagères, et de ne pas exiger de campagne d'éducation auprès du public. Cependant, soulignons que les produits alimentaires d'origine végétale contiennent des substances naturelles qui, à cause de leurs propriétés toxiques, peuvent limiter le potentiel nutritionnel des protéines végétales. Parmi ces substances dites antinutritionnelles se retrouvent les lectines ou phytohémagglutinines (Jaffe, 1980 ; Grant, 1991).

La majorité des travaux concernant l'étude des lectines a été consacrée aux graines de légumineuses (haricot, soja, pois) (Valdebouze et al., 1980; Thorn et al., 1983) et indique que les lectines de ces graines ont des effets négatifs sur l'utilisation digestive et métabolique des protéines (Greer et al., 1985) et des effets toxiques sur les animaux qui les consomment (Rouanet et al., 1985).

Ainsi, dans la perspective d'une exploitation rationnelle de nos graines de courges, il nous paraît judicieux d'entreprendre et d'approfondir les études sur toutes leurs substances antinutritionnelles et notamment leurs lectines.

L'objectif de ce travail est d'examiner la présence de lectines dans les graines de trois espèces de courges Citrullus lanatus, Citrullus sp., Cucumeropsis edulis (Cucurbitaceae) et d'évaluer l'effet du traitement thermique sur ses lectines.

\section{Matériel et méthodes}

\subsection{Matériel}

\subsubsection{Origine et provenance des graines}

Les graines comprennent celles de trois espèces de courges (Cucurbitaceae) Citrullus lanatus (CL), Citrullus sp. (Csp) et Cucumeropsis edulis (CE) appelées respectivement "Bébou", "Wèrè-wèrè" et "N'Viéllè" en langue Baoulé* et aussi celles de haricot, Phaseolus vulgaris (PV) qui servent de référence.

Toutes ces graines sont achetées sur différents marchés du District d'Abidjan, entières et décortiquées.

\subsubsection{Provenance des hématies}

Les hématies utilisées sont issues de sang de lapin provenant d'élevage de ferme et de rat de souche Wistar fourni par l'animalerie du Laboratoire de Nutrition et Pharmacologie, UFR Biosciences, Université de Cocody (Côte d'Ivoire).

\subsubsection{Produits chimiques}

La trypsine est un produit Merck

\subsection{Méthodes}

\subsubsection{Extraction des lectines}

Les graines de courges et de haricot sont broyées au Moulinex et délipidées (courges seulement) avec l'hexane ( $3 \mathrm{~g}$ de graines broyées/ $10 \mathrm{ml}$ d'hexane et le tout agité pendant 1 heure à la température ambiante). La farine obtenue sert 
pour l'extraction des lectines qui se fait avec du tampon phosphate (TP) $\mathrm{pH} 7,4(\mathrm{NaCl} 0,15 \mathrm{M}, \mathrm{KCl}$ $\left.3 \mathrm{mM}, \mathrm{NaHPO}_{4} \mathrm{Na}_{2} \mathrm{PO}_{4} 10 \mathrm{mM}\right)$, selon les méthodes de Budu (1988) et de Cammue et al. (1988). Ainsi $10 \mathrm{~g}$ de farine delipidée ou non est agitée dans $50 \mathrm{ml}$ de TP pendant 1 heure à la température ambiante. Après centrifugation de cette suspension à $10000 \mathrm{trs} / \mathrm{min}$ pendant 10 $\min$ à $4^{\circ} \mathrm{C}$, le surnageant, filtré sur de la compresse de gaze, est recueilli dans un flacon et le culot est repris pour une deuxième extraction. Le surnageant obtenu dans cette dernière est ajouté au premier et le tout conservé à $4^{\circ} \mathrm{C}$, constitue notre extrait brut de protéines.

\subsubsection{Fractionnement protéique}

Le fractionnement protéique est effectué sur $10 \mathrm{ml}$ d'extrait brut de Csp à différentes concentrations de sulfate d'ammonium (pourcentage de saturation): $40 \% ; 60 \% ; 70 \%$; $80 \%$ et $95 \%$; ajoutées par petites fractions appropriées. Le tout est soumis à une agitation mécanique à $0^{\circ} \mathrm{C}$. Après un repos de 1 heure environ, la solution est centrifugée à $10000 \mathrm{trs} / \mathrm{min}$ pendant 20 minutes. Le surnageant obtenu est éliminé et le précipité, recueilli dans du TP et conservé à $4^{\circ} \mathrm{C}$, servira pour les tests d'hémagglutination.

\subsubsection{Traitement thermique}

Le traitement thermique est effectué sur les extrait bruts de CL, Csp, CE et PV.

Trois tubes à essai, contenant chacun une aliquote de l'extrait brut, sont laissés à $100^{\circ} \mathrm{C}$ dans un bain Marie pendant une durée de 15,30 ou 60 minutes. Après le temps requis, l'extrait chauffé puis refroidi à la température ambiante est utilisé pour la détermination de l'activité des lectines.

\subsubsection{Préparation de suspension d'hématies à $4 \%$}

La préparation de suspension d'hématies est effectuée selon la méthode de Higuichi et al. (1988). Le sang de rat ou de lapin est recueilli dans du TP. Le mélange est centrifugé à 4000 trs/min pendant $10 \mathrm{~min}$ à $4^{\circ} \mathrm{C}$ trois fois. Le culot obtenu est dilué avec du TP à raison de $1 \mathrm{ml}$ de culot pour $24 \mathrm{ml}$ de TP, afin d'obtenir une suspension d'hématies à $4 \%$.

A une aliquote de cette suspension d'hématie, le traitement à la trypsine est effectué selon la méthode de Jaffe et al. (1972). Le mélange trypsine - hématie $(0,1 \mathrm{mg}$ de trypsine $/ 10 \mathrm{ml}$ de suspension d'hématie) est incubé à $25^{\circ} \mathrm{C}$ pendant une heure puis centrifugé à 4000 trs/ min pendant 10 minutes à $4^{\circ} \mathrm{C}$. Le culot obtenu est dilué avec du TP à raison de $1 \mathrm{ml}$ de culot pour $24 \mathrm{ml}$ de TP, afin d'obtenir une suspension d'hématies à $4 \%$ traitée à la trypsine.

\subsubsection{Activité hémagglutinante des différents extraits}

L'activité hémagglutinante des lectines est déterminée par la méthode de double dilution sérielle de Jaffe et al. (1972) avec quelques modifications. En effet, $1 \mathrm{ml}$ de chaque extrait (extrait brut ou fraction protéique ou extrait chauffé) est dilué en série avec $1 \mathrm{ml}$ de TP. Ainsi, chaque dilution en série réduit la concentration de l'extrait de moitié.

$40 \mu \mathrm{l}$ de solution mère ou diluée sont mélangés à un volume égal de suspension d'hématie à $4 \%$ traitée à la trypsine ou non dans des puits d'une microplaque. Cette dernière est homogénéisée sur un agitateur pendant 150 min à la température ambiante. Après cette agitation, l'activité hémagglutinante des lectines (teneur en lectines) est déterminée après estimation visuelle de l'hémagglutination. Ces tests sont faits en double (duplicate).

\subsubsection{Expression de l'activité hémagglutinante des lectines}

Cette activité (teneur en lectines) est exprimée en titre qui est la réciproque du plus grand rapport de dilution pour lequel on observe une hémagglutination (Gatner et Podleski, 1975). Soit $1 / 1024$ le plus grand rapport de dilution pour lequel on observe une hémagglutination; le titre équivaut à 1024 qui est la réciproque de 1/1024.

\section{Résultats}

\subsection{Activité hémagglutinante des lectines des extraits bruts.}

L'activité hémagglutinante des lectines des extraits bruts des trois graines de courges est très faible (4 à 32) par rapport à celle de l'extrait brut des graines de haricot (512 à1024). Parmi les trois graines de courges, les extraits bruts de Csp ont l'activité hémagglutinante la plus élevée. Les hématies de lapin sont plus réactives que 
celles de rat vis-à-vis des extraits bruts de CL, Csp et PV. Par contre pour l'extrait brut de CE les réactivités des hématies sont identiques. En outre pour les extraits bruts de Csp et CE, la réactivité des hématies $d$ lapin augmente avec le traitement trypsique. Ce dernier n'a cependant pas d'effet sur les extraits bruts de CL et PV (Tableau 1)

\subsection{Activité hémagglutinante des lectines des fractions protéique de Csp.}

La fraction protéique $\mathrm{F} 5$, obtenue par précipitation au sulfate d'ammonium à $95 \%$ de saturation, présente l'activité hémagglutinante la plus élevée (512). Celle-ci est suivie par ordre décroissant de celles des fractions $60 \%(\mathrm{~F} 2)$; $40 \%(\mathrm{~F} 1) ; 80 \%(\mathrm{~F} 3)$ et $70 \%(\mathrm{~F} 3)$ avec respectivement des titres de $32 ; 8 ; 2$ et 1 . (Tableau 2)

\subsection{Activité hémagglutinante des lectines des extraits bruts chauffés.}

Le traitement thermique des extraits bruts des graines de Csp et $\mathrm{PV}$ à $100^{\circ} \mathrm{C}$ pendant 15 minutes réduit significativement leur activité hémagglutinante qui passe respectivement de 128 à 2 et de 1024 à 16. Lorsque le chauffage atteint 30 minutes, l'activité hémagglutinante de ces deux graines devient nulle.

En revanche, le traitement thermique à $100^{\circ} \mathrm{C}$ pendant 15 minutes est suffisant pour inactiver totalement l'activité hémagglutinante des extraits bruts des graines de CE et CL. (Tableau 3)

Tableau 1: Activité hémagglutinante des lectines des extraits bruts des trois graines de courges et des graines de haricot testés sur les hématies de rat et de lapin (non traitées et traitées).

\begin{tabular}{lccc}
\hline Graines & \multicolumn{3}{c}{ Activité hémagglutinante des lectines (titre) } \\
\hline & Rat & Lapin non traité & Lapin traité \\
$\mathrm{CL}$ & 4 & 8 & 8 \\
$\mathrm{Csp}$ & 8 & 32 & 128 \\
$\mathrm{CE}$ & 4 & 4 & 8 \\
$\mathrm{PV}$ & 512 & 1024 & 1024 \\
\hline
\end{tabular}

CL: Citrullus lanatus; Csp : Citrullus sp.; CE: Cucumeropsis edulis; PV: Phaseolus vulgaris

Tableau 2: Activité hémagglutinante des lectines des fractions protéiques de l'extrait brut de Csp testées sur les hématies de lapin.

\begin{tabular}{cc}
\hline fractions protéiques & Activité hémagglutinante des lectines (titre) \\
\hline F1 & 8 \\
F2 & 32 \\
F3 & 1 \\
F4 & 2 \\
F5 & 512 \\
\hline
\end{tabular}

Csp: Citrullus sp.

F1 : fraction protéique de l'extrait brut de Csp obtenue par précipitation au sulfate d'ammonium à $40 \%$ de saturation.

F2 : fraction protéique de l'extrait brut de Csp obtenue par précipitation au sulfate d'ammonium à $60 \%$ de saturation.

F3 : fraction protéique de l'extrait brut de Csp obtenue par précipitation au sulfate d'ammonium à $70 \%$ de saturation.

F4 : fraction protéique de l'extrait brut de Csp obtenue par précipitation au sulfate d'ammonium à $80 \%$ de saturation.

F5 : fraction protéique de l'extrait brut de Csp obtenue par précipitation au sulfate d'ammonium à 95\% de saturation.

Tableau 3: Activité hémagglutinante des lectines des extraits bruts des graines chauffés à $100^{\circ} \mathrm{C}$ pendant 15,30 ou 60 et testés sur les hématies de lapin traitées à la trypsine (page 8).

\begin{tabular}{cccccc}
\hline & Temps de chauffage (minute) & \multicolumn{4}{c}{ Activité hémagglutinante des lectines (titre) } \\
\hline & & CL & Csp & CE & PV \\
\cline { 2 - 5 } & 8 & 128 & 8 & 1024 \\
30 & 0 & 2 & 0 & 16 \\
60 & 0 & 0 & 0 & 0 \\
& 0 & 0 & 0 & 0 \\
\hline
\end{tabular}

CL: Citrullus lanatus ; Csp : Citrullus sp. ; CE: Cucumeropsis edulis ; PV: Phaseolus vulgaris 


\section{Discussion}

D'une manière générale, l'activité hémagglutinante des lectines des extraits bruts des trois graines de courges est très faible par rapport à celle de l'extrait brut des graines de haricot.

L'activité hémagglutinante des lectines obtenue avec les extraits bruts de nos trois graines de courges est comparable à celle observée par Henderson et al. (1986) sur les variétés des graines de courges des régions arides d'Amérique.

Par ailleurs, les travaux de Henderson et al. (1986) leur ont permis de dégager deux groupes de graines de courges sur la base du niveau faible ou élevé de leur activité hémagglutinante. De même, nos études nous ont permis de classer nos graines de courges en deux groupes: le premier est représenté par les graines de Csp qui ont l'activité hémagglutinante la plus élevée et le second par les graines de CE et CL dont l'activité est faible.

Les hématies de lapin sont plus réactives que celles de rat. Aussi, celles traitées à la trypsine sont elles plus réactives que les non traitées. Cette différence de réactivité des hématies en fonction de l'espèce animale peut s'expliquer d'une part par les spécificités structurales des lectines des différentes variétés de graines (Lis et al., 1985 ; Peumans \& Van Damme, 1995), et d'autre part par le fait que les sites de liaisons des lectines avec les glucides membranaires des hématies pourraient être plus ou moins accessibles selon le type de lectines (Jaffe, 1980). D'ailleurs, le traitement par des enzymes protéolytiques comme la trypsine, la chymotrypsine, la papaïne, la pronase, a pour rôle de digérer les protéines qui masquent les glucides membranaires avec pour conséquence une amélioration de la réactivité des hématies traitées (Jaffe, 1980).

Compte tenu du fait que d'une part, les graines de Csp ont l'activité hémagglutinante la plus élevée de nos graines de courges et d'autre part, les hématies de lapin se sont avérées les plus réactives, ces graines et ces hématies ont été utilisés pour le fractionnement protéique.

La fraction protéique $\mathrm{F} 5$, obtenue par précipitation au sulfate d'ammonium à $95 \%$ de saturation, présente l'activité hémagglutinante la plus élevée
(512). Nos résultats concordent avec ceux de Li (1977) qui a étudié l'activité hémagglutinante des lectines sur les fractions protéiques des graines de Momordica charantia.

Le traitement thermique, appliqué aux différents extraits bruts de nos graines a entraîné une réduction significative de l'activité hémagglutinante de leurs lectines dés la 15è minute, suivie de leur inactivation totale après 30 minutes. Ces observations sont en accord avec celles de Henderson et al. (1986) et laissent donc suggérer que les pratiques culinaires traditionnelles ou modernes des populations contribuent à éliminer les lectines de nos graines de courges et par conséquent à améliorer leur valeur nutritionnelle.

\section{Conclusion}

La présente étude montre que les graines de courges, consommées en Côte d'Ivoire et ailleurs en Afrique, contiennent des lectines; un facteur antinutritionnel. Cependant l'activité hémagglutinante de ces lectines est faible par rapport à celle des graines de haricot de la famille des Légumineuses.

L'effet du traitement thermique indique que les lectines de nos graines de courges sont thermolabiles, ce qui suppose leur inactivation par la cuisson qui, par conséquent, est un moyen efficace pour améliorer leur valeur nutritionnelle.

\section{Références citées}

Aké Assi L., 1984. Flore de la Côte d'Ivoire : Etude descriptive et biogéographique, avec quelques notes ethnobotaniques. Tome IB : Flore des Angiospermes: liste commentée des espèces recensées. (Cecropiaceae à Leguminosae). Doctorat ès Sciences naturelles. Université d'Abidjan, Côte d'Ivoire. 1206 pp.

Budu C. V., 1988. Isolation of two lectins from fir (Abies alba Mill.) bark tissue on immobilized peroxidase and some of their properties. Preliminary study. Rev. roum. Biochim. 25 (1) : 3-7.

Camara F., 1996. Etude du potentiel nutritif des graines de Ricinodendron heudolotii (akpi) et de Citrullus lanatus (wèrè-wèrè). Mémoire de DEA. Université nationale de Côte d'lvoire. 24 pp.

Cammue B., Stinissen W.J., \& Peumans W.J., 1985. Lectins in vegetative tissues of adult barley 
plants grown under field conditions. Plant Physiol. 78: 384-387.

Dos Santos H.K. \& Damon M., 1987 Manuel de nutrition africaine. Eléments de base appliqués. Paris, France : édition Karthala. 308 pp.

Gartner T.K., Podleski F., 1975. Evidence that a membrane bound lectins mediates fusion of $L_{6}$ myoblast. Biophys. Res. Commun. 67: 972-978.

Grant G., 1991. Lectins. In: D'Mello J.P.F., Duffus C.L.M. \& Duffus J.M., Eds. Toxic substances in crop plants. Cambridge: The Royal Society of chemistry. pp. 49-67.

Greer F., Brewer A.C. \& Pusztai A., 1985. Effect of kidney bean (Phaseolus vulgaris) toxin on tissue weight and composition and some metabolic functions of rats. Brit. J. Nutr. 54: 95-103.

Henderson C.W., Scheerens J.C. \& Berry J.W., 1986. Antinutritional factors in Cucurbita seed meals. J. Agric. Food Chem. 34: 434-436.

Higuichi M., Fukumoto Y. \& Iwai K., 1988. Appearance of lectin in winged bean pods during seed development after flowering. J. Agric. Food Chem. 36 (3): 534-536.

Jaffe W.G., 1980. Hemagglutinins (Lectins). In Liener I.E., Eds. Toxic constituents of plant foodstuffs (second edition). New York and London : Academic Press. pp.73-102.

Jaffe W. G., Brucher O. \& Palozzo A., 1972. Detection of four types of specific phytohemagglutinins in different lines of beans (Phaseolus vulgaris). $Z$. Immun, Forsch Bd. 142: 439-447.
Kiki-M'vouaka S., Kouamé K. \& Kamenan A., 1988. Etude de quelques caractéristiques biochimiques et nutritionnelles des graines de cucurbitacées alimentaires de Côte d'Ivoire: activité antitrypsique et digestibilité in vitro des fractions protéiques. Cam. J. Biol. Biochem. Sci. 1 (2): 67-68.

Li S. S-L., 1977. Purification and characterization of seed storage proteins from Momordica charantia. Specialia 33 (7): 895-896.

Lis H., Joubert F.J. \& Sharon N., 1985. Isolation and properties of $\mathrm{N}$-acetyllactosamine-specific lectins from nine Erythrina species. Phytochemistry 24 (12): 2803-2809.

Peumans W.J. \& Van Damme J.M., 1995. Lectins as plant defense proteins. Plant Physiol. 109: 347-352.

Quenum F. \& Kouadio J.B. 1986. Cucurbita edulis (Hook) ou pistache de Côte D'lvoire. Journée Européenne de microscopie et microbiologie des aliments du bétail. 7-9 Juillet 1986. Luxembourg : pp19-21.

Rouanet J.M., Lafont J., Chalet M., Creppy A. \& Besançon P., 1985. Effects of dietary kidney bean (Phaseolus vulgaris) lectins in growing rats. Nut. Rep. Int. 31 (1): 237-244.

Thorn K.A., Tinsley A.M., Weber C.W. \& Berry J.W., 1983. Antinutritional factors in legumes of the Sonaran desert. Ecol. Food Nutr. 13: 251-256.

Valdebouze P., Bergeron E., Gaborit T. \& DelorsLaval J., 1980. Content and distribution of trypsin inhibitors and hemagglutinins in some legume seeds. Can. J. Plant Sci. 60 : 695-701 